\title{
EL ACUERDO DE \\ PRINCIPIO DE \\ SEPTIEMBRE DE \\ 1993 ENTRE \\ ISRAEL Y LA OLP:
}

ESQUEMA CONTEXTUAL

Kenza Elmandjra*

A la memoria de Johan Jorgen Holt

$\mathbb{A}_{1}$

Acuerdo de Principio firmado por

el Primer Ministro Israelí, Isaac Rabin, y el Jefe de la Organización para la Liberación de Palestina (OLP), Yasser Arafat, el día 13 de septiembre de 1993 en el jardín de la Casa Blanca en Washington se caracterizó por mucha pompa. El evento fue transmitido a todo el mundo con la certeza que brindaba una nueva era de paz y de entendimiento en el Medio Oriente. ${ }^{1}$ En su discurso, durante la ceremonia, el Presidente William Clinton se refirió constantemente a la santidad de la región entre el río Jordan y el mar Mediterráneo donde se debe lograr la paz, hasta comparar a Rabin y Arafat con los hijos de Abraham, Isaac e Ishmael. En su intervención, el Ministro de Relaciones Exteriores de Israel, Shimon Peres, se refirió a la tierra sagrada y a la Ciudad de Jerusalén como

* Departamento de Estudios Internacionales, I.T.A.M.

${ }^{1}$ Los medios de comunicación no perdieron la oportunidad de señalar la renuencia de Rabin a estrechar la mano de Arafat cuando éste se la extendió. 
Kenza Elmandjra

símbolos de que "Abraham tenía fe en la libertad". ${ }^{2}$ En general (aparte de los de Yasser Arafat y Mahmoud Abbas, el encargado de asuntos políticos de la OLP), los discursos adoptaron un lenguaje casi religioso, como si al firmar el Acuerdo todos estuvieran respondiendo a la voluntad de Dios y no a la realidad política, militar y económica de esa turbulenta región del mundo.

Se ha dicho que este Acuerdo fue posible en gran parte a causa de los cambios políticos internacionales que resultaron del fin de la Guerra Fría. Pero las razones de dicho Acuerdo son más complejas y sería una simplificación subrayar una sola causa. Las variables causantes fueron múltiples, interrelacionadas y complejas: cambios sistémicos como el fin de la bipolaridad, la Guerra del Golfo y sus efectos; cambios internos como un nuevo gobierno laborista y la crisis económica en Israel, la Intifada y la pérdida de legitimidad internacional e interna por parte de la OLP. A éstas se podrían agregar el fracaso de las negociaciones en Washington entre Israel y la delegación palestina, y el papel de mediación de Noruega, entre otras. Como lo indicó la entonces vocera de la delegación palestina en las negociaciones de Washington, Hanan Ashrawi, "el Acuerdo ocurrió en una coyuntura especial. Una de estas coyunturas que sucede raramente y que se debe aprovechar ". 3

Para dar una explicación completa se requiere de una información completa, lo que en este caso es muy difícil de lograr por la naturaleza de las negociaciones y por el hecho que el proceso de éstas no ha terminado. No obstante, para no caer en pura especulación, se ofrece una esquema contextual del proceso que llevó al Acuerdo. En la primera parte, se exponen los puntos básicos del Acuerdo de Principio de septiembre de 1993. En las dos siguientes partes, se analizan las causas e intereses que motivaron a Israel y a la OLP a negociar entre ellos y llegar a dicho acuerdo. Finalmente, a modo de conclusión, se abordarán los posibles límites del Acuerdo así como las dificultades de aplicación que se podrían presentar en el futuro.

${ }^{2}$ Para los discursos del Presidente Clinton, Shimon Perez, Mahmoud Abbas, Yitzhak Rabin y Yaser Arafat, ver The New York Times, 14/IX/93, p. A-12.

${ }^{3}$ Comentario hecho a Ted Kopel, en el programa Nightline, de la cadena norteamericana $\mathrm{ABC}, 11 / \mathrm{IX} / 93$. 
ACUERDO ENTRE ISRAEL Y LA OLP

\section{El acuerdo de principio}

\section{El concepto de autonomía limitada}

Antes de dar una breve descripción del Acuerdo de Principio es importante destacar lo que éste no es: ni un tratado ni un acuerdo de paz; es un acuerdo político limitado. Como lo indica su propio nombre es un "Acuerdo de Principio" entre Israel y la OLP, basado en un reconocimiento mutuo y que esboza el proceso de otorgamiento de una "autonomía limitada" a Gaza y Jericó. Este proceso podría conducir en el futuro a la formación de un Estado Palestino independiente.

El Acuerdo no llama a la creación de un Estado Palestino independiente con fronteras propias, soberano y reconocido internacionalmente, sino que trata de una "autonomía limitada", que está lejos de las aspiraciones independentistas. Era lo único que Israel estaba dispuesto a otorgar; lo único que los palestinos podían pedir y obtener. La naturaleza vaga del Acuerdo fue una decisión consciente de ambas partes. Los dos equipos negociadores acordaron limitar dicho acuerdo a una serie de principios para, una vez que se hiciera público, comenzar las negociaciones sobre aspectos sustantivos. Como lo indicó el Ministro de Relaciones Exteriores israelí, Shimon Peres, en una conversación con el Secretario de Estado norteamericano, Warren Christopher, un poco antes de la firma del Acuerdo, "... si uno piensa en todos los detalles, se paraliza ". 4

\section{"Gaza y Jericó primero"}

"Autonomía limitada" se llamó en el Acuerdo al otorgamiento a palestinos de la autoridad para manejar los asuntos de educación, cultura, salud, bienestar social, impuestos directos y turismo. Esta autoridad tendrá que ser transferida de Israel a "palestinos autorizados" de Gaza y

${ }^{4}$ The New York Times, 5/IX/93, sec. 4, p. 1. 
Kenza Elmandjra

Cisjordania. Israel retendrá el control sobre las relaciones exteriores y los asuntos de seguridad.

Geográficamente, el alcance de esta autonomía limitada se restringirá a la franja de Gaza y la Ciudad de Jericó, por eso es conocido como el plan "Gaza y Jericó primero". Gaza ocupa una extensión de 40 km. de largo y 10 de ancho, donde viven 700 mil palestinos; uno de los lugares del mundo con mayor la densidad de población: 1,75 habitantes por metro cuadrado. En la Ciudad de Jericó - una de las más antiguas del mundo, con más de 8,000 años- actualmente viven 15,000 palestinos. ${ }^{5}$ Gaza se sitúa geográficamente entre Israel, Egipto y el mar Mediterráneo, pero su frontera con Israel queda por definirse, dada la presencia de colonias judías. Los límites de Jericó también están por definirse. Para los palestinos comprende toda la región histórica, con una superficie de $345 \mathrm{~km}$. cuadrados, mientras que Israel los limita a la ciudad y tres campamentos de refugiados palestinos, en un área de $25 \mathrm{~km}$. cuadrados. ${ }^{6}$ Esta contradicción es importante porque podría frenar la aplicación del Acuerdo. ${ }^{7}$

\section{Instrumentación: caminos y desvíos}

En términos operativos, la autonomía limitada se otorgará en cuatro etapas. Primero, se formará una fuerza policíaca cuyos efectivos serán miembros de la OLP que viven fuera de los territorios ocupados. Segundo, se formará un comité de enlace palestino-israelí para negociar los detalles de la instrumentación. Tercero, se formará otro comité palestino-israelí para discutir asuntos económicos, como el agua, la electricidad, la energía, las finanzas, el transporte, la comunicación, las relaciones laborales, la capacitación, la protección del medio ambiente y los medios de información. Y cuarto, Jordania y Egipto tendrán que unirse a las

${ }^{5}$ Le Monde Diplomatique, Vol. 39, no. 454, I/92, p. 22; e Instituto Noruego de Ciencias Sociales Aplicadas, Centro para el Análisis de Palestina (1993), citado en The New York Times, 14/IX/93, p. A-9.

${ }^{6}$ El Financiero, $16 / \mathrm{XI} / 93$, p. 65.

${ }^{7}$ Las dificultades frente a la imposibilidad de aplicar el plan el 13 de diciembre de 1993, como estaba previsto, lo confirman. 
comisiones mixtas para discutir el regreso de los palestinos desplazados de Cisjordania y Gaza después de la Guerra de 1967, que se estiman en 800,000 , incluyendo a sus dependientes. ${ }^{8}$

El Acuerdo también esboza una cronología respecto a su aplicación y programa. Cinco fechas están enunciadas: El 13 de diciembre de 1993, israelíes y palestinos tenían que haber firmado un acuerdo sobre el retiro del ejército israelí de Gaza y Jericó, y transferir el control a los palestinos autorizados. A partir de esta fecha tenía que empezar el período interino de autonomía palestina que duraría cinco años. El 13 de abril de 1994 es la fecha límite para el cumplimiento del retiro isrealí de Gaza y Jericó. El ejército israelí se retirará de las zonas pobladas palestinas pero no de los asentamientos judíos de los alrededores de Gaza y Jericó. Además tendrán el control de la frontera entre Gaza y Egipto, y el puente Allenby, cerca de Jericó, que comunica Cisjordania con Jordania. El 13 de julio de 1994 es la fecha límite para la elección de un Consejo Palestino que tendrá poderes políticos para manejar los asuntos internos de la Franja y la Ciudad. No está precisado si los palestinos de Jerusalén del Este podrán formar parte de dicho Consejo. En este momento las fuerzas militares israelíes se retirarán y scrán desplegadas en las afueras para garantizar la seguridad de los colonos judíos. A partir de entonces el gobierno militar israelí será eliminado y la administración civil israelí disuelta. El 13 de diciembre de 1995 es la fecha limite para concluir las negociaciones sobre un Acuerdo Final. En dicho acuerdo se tendrán que resolver asuntos relacionados al estatus de Jerusalén, los refugiados palestinos, los asentamientos judíos y las fronteras entre Israel y la entidad palestina. El 13 de diciembre de 1998 tendrá lugar el Acuerdo Final, que culminará lo iniciado cinco años antes con el Acuerdo de Principio (1993): la creación de un Estado Palestino independiente. ${ }^{9}$

\section{Los intereses del Estado de Israel}

El más importante interés nacional de Israel siempre ha sido su seguridad territorial. En 1993, el reconocimiento de la OLP como único repre-

\footnotetext{
${ }^{8}$ Para un resumen del Acuerdo, ver The New York Times, 14/IX/93, p. A-8.

${ }^{9}$ Ibid.
} 


\section{Kenza Elmandjra}

sentante del pueblo palestino y —más importante aún- la aceptación del derecho político del pueblo palestino, así como la voluntad de negociar el Acuerdo del 13 de septiembre de 1993 a pesar de que podría significar una pérdida de control de partes de la zonas ocupadas, fue vista como la mejor estrategia israclí para proteger y mantener su seguridad nacional. La razón principal del Acuerdo se buscaba en términos de su contenido y aplicación. ${ }^{10}$

Frente a una fuerte crisis económica agudizada por la migración masiva de judíos rusos a partir de 1990 , Israel tenía que llegar a una solución en cuanto a los territorios ocupados. ${ }^{11}$ Ésta, por una parte, debía reducir sus gastos (posiblemente acudiendo a una mayor ayuda económica extranjera) $y$, por otra, mantener la seguridad territorial de la nación. A partir de 1991, se buscó este tipo de acuerdo y se concluyó que la mejor manera de conseguirlo era negociarlo con los palestinos. A continuación, se analizarán las prerrogativas estratégicas de Israel en cuanto a los territorios ocupados. Posteriormente, se examinará porqué Israel decidió romper con su tradicional postura y negoció directamente con los palestinos y la OLP. En la última parte se ilustrará este análisis con referencia específica al caso de Gaza.

${ }^{10}$ Se ha escrito que el Acuerdo fue el resultado directo del fin de la bipolaridad. Supuestamente las presiones norteamericanas sobre la necesidad de Isracl de justificar su ayuda económica por el fin de la Guerra Fría, y la supuesta pérdida del interés estratégico de esta nación, fueron claves. Pero es importante reconocer que Israel por su cercanía con la zona petrolera del Golfo Pérsico, sigue siendo de un interés estratégico primordial para los Estados Unidos, Europa y Japón.

${ }^{11}$ A una pareja judía rusa con un niño que emigra a Israel, el gobierno israelí le otorga inmediatamente US $\$ 9,000$. Reciben además una carta de identidad y el derecho de voto. Un problema que se ha presentado es que aproximadamente $30 \%$ de los rusos que llegaron no eran judíos. Se estima que entre enero de 1990 y septiembre de 1990 llegaron a Israel más de 300.000 rusos judíos ( 7.000 durante el mes de febrero de 1990), cifra que se dobló entre 1991 y 1993. Le Monde Diplomatique, vol. 38 , no. 451 , oct. 91 , p. 20-1. 


\section{Prerrogativas estratégicas de Israel}

Israel ocupa desde la guerra de 1967 a Cisjordania (incluyendo la Ciudad de Jerusalén), la Franja de Gaza y el Sinaí (devuelto a Egipto en 1979). En 1973 ocupó el Golán, que pertenecía a Siria, anexado oficialmente en 1981. En 1985, tres años después de su invasión a Líbano, las fuerzas militares israelíes ocuparon la denominada "zona de seguridad", al sur de Líbano. La ocupación de dichos territorios presenta a Israel ventajas y costos.

En términos de seguridad nacional estos territorios significan para Israel un campo de acción más grande, que le permite una mayor estrategia defensiva y ofensiva. Por la presencia militar en esas zonas, se obtiene una mayor vigilancia y seguridad del territorio del Estado y de sus principales ciudades, como Tel Aviv; así como una mayor cercanía a los Estados de Líbano, Siria, Jordania y Egipto, para poder actuar rápidamente en caso de amenazas militares. Dichas zonas, además, ofrecen recursos económicos indispensables para la sobrevivencia de Israel. No sólo favorecen la expansión de las colonias judías, sino que también concentran la mayoría de sus recursos hidráulicos y tierras arables. Por ejemplo, Israel obtiene el $65 \%$ de su agua fresca renovable de dos mantos acuíferos, uno situado bajo la Franja de Gaza y el otro cerca de la costa del Mediterráneo. El resto de su agua proviene del Río Jordán y su cuenca de almacenamiento en Cisjordania. ${ }^{12}$

Pero, junto con las ventajas que ofrece la ocupación de los territorios, se presentan desventajas, principalmente los costos políticos y económicos que implica el control de la población palestina. Sobre todo, desde el principio de la "Intifada" o "la guerra de las piedras" de los jóvenes palestinos en noviembre de 1987 , los costos han aumentado drásticamen-

${ }^{12}$ En el Golán, se encuentra el 35\% de las reservas de agua de Israel. En Cisjordania, los pozos profundos construidos por los colonos judíos secan los menos profundos de los palestinos. El Río Linati en el sur del Líbano, siempre ha sido una tentación, que la "zona de seguridad" ha concretado. Para más datos, referirse a Pritt J. Vislind, "The Middle Easts Water: Critical Resource", National Geographic, vol. 183, no. 5, mayo 93, p. 38-71. 


\section{Kenza Elmandjra}

te. En términos económicos, comprenden los gastos militares de las fuerzas de seguridad para sofocar la rebelión y preservar la seguridad de los colonos judíos y las vías de comunicación. En términos políticos, los costos consisten en las críticas internas e internacionales por violaciones de derechos humanos debido al uso de la fuerza militar y la detención de palestinos sin que proceda un proceso judicial. A nivel internacional, eso implica el riesgo de perder parte de la ayuda económica extranjera recibida por Israel.

La mejor solución era entonces llegar a un cierto acuerdo, por el que se pudiera mantener una presencia militar israelí en los territorios en cuestión y en la mayoría de los asentamientos de colonos, un cierto control sobre los recursos naturales de los territorios (principalmente agua) y, al mismo tiempo, acabar con la rebelión palestina. El precio a pagar podría incluir el desmantelamiento de ciertas colonias judías y una mayor autonomía palestina, que eventualmente se concretaba en un Estado Palestino independiente.

Cambio de estrategia de negociación de Israel: negociar directamente con los palestinos

Al principio del mes de septiembre de 1993, cuando se hicieron públicas las negociaciones directas entre altos funcionarios israelíes y palestinos de la OLP, el Primer Ministro israelí Isaac Rabin comentó: "Estoy puesto a hacer dolorosos compromisos. La paz no se hace con amigos. La paz se logra con enemigos, a uno de ellos - y no voy a mencionar nombreslo aborrezco mucho." 13

Más allá de la simple lógica del argumento que "la paz se hace con enemigos", el hecho de que escogieran a la OLP y a Yasser Arafat como interlocutores es altamente ilustrativo. Por primera vez desde la creación de Israel en 1948, el gobierno israelí estaba decidido a llegar a un compromiso directamente con los palestinos. A fines de 1991 optó por abandonar su tradicional estrategia de no considerar a éstos y negociar sólo y bilateralmente con cada nación árabe para llegar a un entendimiento sobre la seguridad territorial de Israel. Esta táctica obedecía a

${ }^{13}$ The New York Times, 5/IX/93, sec. 4, p. 1. 
que rompiendo el frente árabe, los israelíes incrementaban su poder de negociación obteniendo mayores concesiones sin tener que tomar en cuenta las aspiraciones independentistas palestinas. El ejemplo más claro es el Acuerdo de "Camp David" de 1979, con Egipto, por el que Israel devolvió el Sinaí a Egipto, a cambio de un pacto mutuo de no agresión. El trato de la cuestión palestina se hizo sin la consulta de dicho pueblo y únicamente se limitó a un acuerdo sobre un período de transición de cinco años para una autonomía limitada de los territorios ocupados.

Después de la Conferencia de Madrid de octubre de 1991, empezaron en Washington negociaciones directas entre Israel y representantes palestinos. Entre diciembre de 1991 y agosto de 1993, hubo once rondas de negociación; pero cada una acabó con un fracaso casi total. Las razones fueron múltiples. Una de ellas fue que los únicos palestinos autorizados a participar en las negociaciones eran de los territorios ocupados, sin ninguna relación (oficial) con la OLP. Debido a que los negociadores palestinos no contaban con la capacidad política para aplicar los acuerdos, los israelíes empezaron a percibir estas negociaciones como inútiles. No obstante, es muy difícil reconocer si desde el principio Israel no practicaba el doble juego de una actitud conciliadora (negociaciones directas con los palestinos) y conflictiva (rechazo de compromisos). Lo importante es que por primera vez entre 1991 y 1993 los palestinos, a través de la delegación en las negociaciones de Washington, fueron reconocidos como interlocutores. La nueva estrategia de Israel fue seguir con sus negociaciones bilaterales pero ahora con los palestinos.

Por el fracaso de dichas negociaciones y el deseo de llegar a un compromiso que garantizara a Israel su seguridad nacional, el gobierno de Isaac Rabin entendió que necesitaba un acuerdo aplicable que respondiera a las prerrogativas económicas y estratégicas de Israel. La OLP se presentó entonces como el único interlocutor palestino con quien se podría llegar a un acuerdo de esa naturaleza.

\section{Negociar directamente con la OLP}

Los israelíes estaban listos no sólo para negociar con los palestinos sino también —más importante- directamente con la OLP, una organiza- 


\section{Kenza Elmandjra}

ción tradicionalmente calificada de "terrorista". Los términos del Acuerdo de septiembre de 1993, con el reconocimiento mutuo Israel-OLP, representan un cambio radical de la postura israelí en el conflicto del Medio Oriente. Dos explicaciones se pueden ofrecer en cuanto a porqué escogieron a la OLP como interlocutor.

La primera tiene que ver con la pérdida de confianza en las negociaciones de Washington. A juicio de los negociadores israelíes era importante hablar con los que tenían el poder político para obtener cualquier compromiso. Para el gobierno israelí, las alternativas eran tres. Una, continuar las negociaciones de Washington y no llegar a nada. Dos, dialogar con otras organizaciones palestinas como el grupo Hamas o el Frente Popular para la Liberación de Palestina. (Por la naturaleza "extremista" de estos grupos, a juicio de Israel, esto era imposible.) Y tres, romper toda negociación con los palestinos y permanecer en el status quo. Pero, como hemos señalado, estaba en el interés de Israel buscar un acuerdo en cuanto al futuro de los territorios ocupados, y para ello quién mejor que la OLP.

La segunda explicación es que a pesar del tradicional rechazo a dialogar con él, los israelíes sabían que escogiendo a Yasser Arafat y la OLP, no sería difícil llegar a un compromiso que respetara los intereses primordiales de Israel. La OLP, como se destacará en la siguiente sección, era percibida como el mejor interlocutor. Los israelíes tenían plena conciencia de las múltiples debilidades de ésta en cuanto a su pérdida de credibilidad internacional y de control sobre la comunidad palestina y sus problemas financieros. Aprovechando estas dificultades, Israel al acercarse a la OLP le proponía una actuación capaz de fortalecerla ante dichas debilidades y restaurar su credibilidad. Por su lado, Israel incrementaba su poder de negociación y la probabilidad de obtener concesiones.

\section{Ilustración: deshacerse de Gaza}

Los crecientes disturbios en los territorios ocupados y principalmente en Gaza, además de la perspectiva de una revigorización de la Intifada después de la relativa calma durante la Guerra del Golfo (1990/91), se percibían en Israel como elementos de alto riesgo para su seguridad 
territorial. Además y aún más importante, los altos costos de mantener el orden en los territorios empezaban a sentirse, sobre todo con la llegada de miles de judíos rusos que debilitaban la economía del Estado. El arreglo fue transferir la responsabilidad de la seguridad en Gaza y Jericó a fuerzas palestinas "autorizadas" por los israelíes. Quién mejor que los mismos palestinos para controlar la creciente "ola islámica" y el recrudecimiento de los disturbios en Gaza.

Con esta transferencia de autoridad, la OLP se compromete a garantizar el orden y la seguridad en Gaza, mientras que Israel, no sólo baja los costos que esto implica, sino que también adquiere dos ventajas resultantes. La primera es relativa a su imagen internacional; no serán soldados israelíes los acusados de maltratar, lesionar y matar a los "niños de las piedras" de la Intifada, sino los propios palestinos. Eso puede redundar en mayor apoyo financiero, principalmente de los Estados Unidos, con una aprobación más rápida de su órgano legislativo. La segunda ventaja es que si la OLP no impide ataques a colonos judíos por parte de palestinos, se planteará la necesidad de mantener una presencia militar israelí en la zona. Como lo destacó Mordejai Gur, Vice Ministro de Defensa israelí, Israel podrá intervenir en "cualquier rincón de Gaza y Cisjordania" si el gobierno autónomo palestino "no puede controlar a los terroristas". 14

\section{Los intereses de la OLP}

Considerando la fuerte oposición al Acuerdo con Israel que surgió dentro de la OLP, se tendría que hablar más de los intereses del propio Yasser Arafat y de sus más cercanos colaboradores, que de los de la OLP, para poder entender la dimensión política de las negociaciones con Israel sobre una autonomía limitada. En efecto, los desacuerdos dentro de la OLP se manifestaron inmediatamente después del anuncio de las negociaciones secretas y del Acuerdo de Principio. ${ }^{15}$ Al día siguiente del

${ }_{15}^{14}$ La Jornada, 1/IX/93, p.54.

${ }^{15}$ Una razón por la cual las ncgociaciones fueron secretas fue justamente la previsión por parte de Yasser Arafat y colaboradores de un rechazo por parte de algunos miembros de la OLP, como del grupo Fatah y del CNP. 
Kenza Elmandjra

anuncio del Acuerdo, el Presidente del Consejo Nacional Palestino (CNP), el Parlamento Palestino en el exilio, Nabih Berri, lo condenó. ${ }^{16}$ El más serio desacuerdo se dió dentro del Comité Ejecutivo del CNP donde solamente 8 de los 18 miembros votaron a favor del Acuerdo. ${ }^{17}$ De los más destacados luchadores por la causa palestina, el poeta Mahmoud Darwish renunció; Shafik al-Hut, el representante de la OLP en Libano suspendió su participación; y Faruk Kaddumi, el co-fundador del grupe Fatah no asistió a la reunión. ${ }^{18}$ En diciembre de 1993, la vocera de la delegación palestina en Washington, Hanan Ashrawi renunció, supuestamente por su desacuerdo con las posiciones de la OLP en las negociaciones sobre los detalles de aplicación del Acuerdo de septiembre de $1993 .^{19}$

Para explicar porqué la OLP estaba dispuesta a negociar directamente con Israel y llegar a un acuerdo que sería altamente condenado dentro de la organización y que no le aseguraba un camino directo a la creación de un Estado palestino independiente, primero estableceré el contexto histórico del reconocimiento de Israel por parte de la OLP. En las siguientes dos partes, se analizarán los intereses de la OLP para llegar a un acuerdo tan limitado con Israel. Éstos serán divididos en externos, como el deseo de la OLP de restablecer su credibilidad internacional y asegurar una presión internacional sobre Israel para que cumpla con los términos del Acuerdo; e internos, principalmente el deseo de controlar su propio destino y el futuro de la comunidad palestina.

${ }^{16}$ La Jomada, 15/IX/93, p. 1.

${ }^{17}$ En las elecciones generales, 63 votaron a favor, 8 en contra y 9 se abstuvieron. La Jomada, 12/X/93, p. 54. En el CNP, los 8 que votaron a favor fueron: Yasser Arafat, Abu Mazen, Yasser Ared Raboo, Mohammad Zohbi Al-Nachachgibi, Yasser Amro, Jawad Al-Ghossaim, Samir Goche y Soleiman Najjad. Cabe mencionar que Elia Khoury no participó por razones de salud. Mahdi Elmandjra, "La Paix! Quelle Paix?" Libération, Marruecos, 1/X/93, p. 6.

${ }^{18} \mathrm{Ibid}$; y Edward W. Said, "Comment Conjurer le Risque dune Perpétuelle Soumission à lÉtat dIsraël", Le Monde Diplomatique, vol. 40, no. 476, nov. 93, p. 4-5.

${ }^{19}$ El Financiero, 11/XII/93, p. 21. 


\section{El reconocimiento del Estado de Israel}

En diciembre de 1987, en un campo de refugiados de Gaza empezó la denominada "Intifada", que en lengua árabe significa "desbordamiento". Como lo indicó Edward Said, "la Intifada no encarnaba el terrorismo y la violencia, pero sí el derecho a resistir". ${ }^{20}$ Este derecho a resistir de los palestinos que viven en los territorios ocupados, fue ejercido sin pedir permiso a la OLP, por lo tanto actuando independientemente de cualquier orden. La Intifada es un acto sui generis de los palestinos de los territorios ocupados, hartos de la presencia militar israelí. La OLP trató entonces de poner su propio sello sobre la Intifada para probar que los líderes de ésta y la dirigencia de la OLP estaban ligados; y para asegurarse cierto control de los acontecimientos en los territorios.

Las resoluciones votadas por el CNP en Argelia en noviembre de 1988, la Declaración de Independencia y la elección de Yasser Arafat como Presidente de Palestina fueron medidas para acapararse los actos de los palestinos en los territorios ocupados. En las asambleas del CNP, la Resolución 242 (1967) del Consejo de Seguridad de las Naciones Unidas fue declarada como la única solución para llegar a una acuerdo con Israel. Por este hecho, implícitamente la OLP reconoció el derecho de Israel a existir como Estado. Un mes después, en diciembre de 1988, Yasiser Arafat leyó una declaración donde lo reconoció oficialmente, haciendo un llamado a la renuncia de "actos terroristas" fuera de los territorios ocupados. ${ }^{21}$ En otras palabras, el interés por parte de la OLP de abrir un diálogo directo con Israel no era nuevo. En 1993, la novedad era la disponibilidad de la OLP para hacer concesiones en cuanto al estatus de autonomía limitada, que siempre había sido rechazado como solución.

${ }^{20}$ Said, "Comment Conjurer ...", p. 4. Edward Said, profesor de literatura inglesa en la Universitad de Columbia en Nueva York, fue miembro del Consejo Nacional de la OLP de 1977 a 1991.

${ }^{21}$ Alain Gersh, "Enfin, La Paix?", Le Monde Diplomatique, vol.38, no. 452, nov. 91 , p. 1 y 3. 
Kenza Elmandjra

\section{Factores externos: credibilidad internacional y presión sobre Israel}

Por primera vez en su historia, la OLP fue reconocida por Israel como actor en lo que siempre se había llamado el conflicto Arabe-Israelí. La OLP, fundada en 1964 en El Cairo, fue reconocida en 1974 por la Asamblea General de las Naciones Unidas como "el único y legítimo representante del pueblo palestino", recibiendo el estatus de miembro observador en dicha organización. Pero, desde su desastrosa estrategia durante la Guerra del Golfo, cuando fue acusada de alinearse con el régimen de Sadam Hussein, la OLP perdió mucho prestigio a nivel internacional. (Es importante reconocer que en sus declaraciones, la OLP condenaba el uso masivo de la fuerza por parte de Estados Unidos y sus aliados. Como víctima misma de invasión y ocupación, la OLP no podía aprobar la invasión iraquí de Kuwait.) Una de las consecuencias de dicha estrategia fue la drástica reducción del apoyo financiero por parte de los países del Golfo, que se sumó a los gastos por ayuda económica que la OLP proporcionó a miles de palestinos expulsados de esos países. ${ }^{22}$

74 Por ello, al entablar negociaciones directas con Israel y lograr un acuerdo, aunque limitado, pretende restablecer su credibilidad en la escena internacional, además de ejercer un control directo sobre el futuro de los palestinos asentados en los territorios ocupacios y en otros países. Asimismo, las negociaciones directas con Israel demostrarían a las demás naciones la seriedad de la OLP, dado su deseo de resolver el conflicto por la vía pacífica y aumentaría su respeto en los foros internacionales, con efectos políticos y económicos positivos. En cuanto a efectos políticos, el Acuerdo llevó a la reanudación de sus relaciones con los Estados Unidos en octubre de $1993 .{ }^{23}$ Estas relaciones, iniciadas en 1988, fueron interrumpidas durante la Guerra del Golfo a raíz de la posición que adoptara la organización. Lo importante del restableci-

${ }^{22}$ Ver, Georges Corm, "L’Aggravation des Déséquilibres et des Injustices Économiques au Proche Orient", Le Monde Diplomatique, vol. 40, no. 474, sept. 93, p. $14-5$.

${ }^{23}$ La Jomada, 16/X/93, p. 52. 
miento es que son cruciales para afianzar su autoridad como representante del pueblo palestino, y crear un medio de presión frente a Israel para que cumpla con los términos del Acuerdo. Como lo demuestran las gestiones de mediación del entonces Secretario de Estado Henry Kissinger y del Presidente Jimmy Carter en 1973/74 para los Acuerdos Interinos entre Israel, Siria y Egipto al final de la guerra de 1973, y en 1978/79 con el Acuerdo de Campo David, Estados Unidos fue el único país con recursos e influencia sobre Israel para conseguir que éste negociara de buena fe y cumpliera con sus compromisos. Sin el apoyo de los Estados Unidos, la OLP sabía que carecía de un medio de presión necesario para que Israel cumpla con los términos del Acuerdo.

En cuanto a los efectos económicos, el Acuerdo le abriría a la OLP contactos con la comunidad internacional y posiblemente ayuda financiera por parte de ciertos países de la Comunidad Europea o Japón. Además, con una posición moderada, los flujos financieros de los países del Golfo Pérsico se podrían reanudar. En octubre de 1993, además de reiniciar relaciones con la OLP, el Senado norteamericano suspendió "temporalmente" las sanciones que impedían a la OLP acceder a la asistencia económica de los Estados Unidos a través de organismos internacionales. ${ }^{24}$ Pero, al no cumplirse la primera fase del Acuerdo el 13 de diciembre de 1993, no se otorgó la ayuda económica prometida por el Banco Mundial, la Comunidad Europea y los Estados Unidos para apoyar al Acuerdo de septiembre de 1993 . $^{25}$

Es importante señalar que estos efectos no se lograron con las declaraciones de 1988 ni con las negociaciones de Washington. En 1988, Israel no tenía interés en llegar a un acuerdo directo con la OLP; por otro lado, las negociaciones de Washington fueron un fracaso. ${ }^{26}$ En efecto, desde que empezaron éstas, la OLP había dado el visto bueno a las mismas, tratando de cambiar el estatus de la Organización, de simple observador

\section{${ }^{24}$ Ibid.}

${ }^{25}$ El Banco Mundial calculó en 3,000 millones de dolares la ayuda "urgente" necesaria para apoyar el proyecto Gaza-Jericó en la próxima década, The New York Times, 12/IX/93, 1 $1^{\text {a }}$ sec., p. 11.

${ }^{26}$ Las negociaciones comprendían principalmente asuntos técnicos como el agua y la electricidad en los territorios ocupados, sin tocar los temas centrales como el futuro político de esos territorios, los asentamientos judíos o la presencia militar israclí. 
Kenza Elmandjra

a participante activo. La oferta de negociar directamente con Israel, hecha por los mediadores noruegos, fue bienvenida. Además, para la delegación palestina compuesta por palestinos de los territorios ocupados sin lazos oficiales con la OLP, las negociaciones fueron percibidas como un fracaso por dos razones interrelacionadas. Primero, los israelíes parecían decididos a no hacer ninguna concesión; y segundo, a juicio de los palestinos, tampoco actuaban de buena fe. Hanan Ashrawi, entonces vocera de la delegación palestina en Washington, declaró que "después de 14 meses, las negociaciones no registraron avance alguno". ${ }^{27}$ Como lo indicó también Abdul al-Amid al-Saih, cuando renunció a su cargo de jefe de dicha delegación después del fracaso de la décima ronda, "lo que [Israel] nos está imponiendo es una capitulación, no la paz". ${ }^{28}$ En vista de que no se llegó a resultado alguno, y de que la OLP percibió el deseo de Israel de negociar con ellos, aceptaron abrir negociaciones secretas.

\section{Factores internos: la OLP como único representante del pueblo pa- lestino}

Días después de dar la mano al Primer Ministro de Israel, Isaac Rabin, Yasser Arafat, Jefe de la OLP y líder del grupo mayoritario Fatah, declaró: "No quiero ser otro Gorbachev. No soy un Gorbachev." ${ }^{29}$ Esta declaración es muy significativa porque subraya algunas razones por las que él y su organización estaban dispuestos a reconocer oficialmente a Israel y a firmar un acuerdo limitado. Arafat expresaba que de modo semejante a como sucedió con Gorbachev, podría perder no sólo el control de la OLP, sino también del proceso político en los territorios ocupados con respecto al futuro de la comunidad palestina. Pero sus intereses y los de sus colaboradores de negociar el Acuerdo con Israel, más que a la "pesadilla gorbachoviana", respondían a la creciente pérdida de control de la OLP sobre la comunidad palestina, y con ello del futuro político de ésta.

${ }^{27}$ Entrevista en L'Express, París, 14/I/93, p. 68-71.

${ }^{28}$ La Jornada, 23/V/93, p. 52.

${ }^{29}$ The Washington Post, 15/IX/93, p. 1. 
Esta pérdida de autoridad sobre la comunidad palestina empezó a principios de los 80 y puede explicarse a través de dos importantes eventos. En Líbano, la OLP siguió una pésima estrategia; tratando de acreditarse los triunfos falangistas durante la guerra civil, se convirtió en el blanco de las fuerzas militares israelíes, que forzaron a su dirigencia a huir a Túnez después de la invasión israelí en junio de 1982. El segundo evento fue la Intifada, que empezó en diciembre de 1987 con el levantamiento de un campo de refugiados palestinos en Gaza. A principios de 1993, con la revigorización de la Intifada y la creciente popularidad de otras organizaciones palestinas, la OLP entendió que necesitaba actuar para restaurar su control en los territorios ocupados.

El crecimiento de la popularidad de otras organizaciones palestinas en los territorios ocupados (algunas afiliadas con la OLP) preocupaba a la dirigencia de la OLP. Como comentó antes de la firma del Acuerdo de 1993 el vocero del grupo Hamas, Ibrahim Goshe, referiéndose a grupos como el Frente Democrático para la Liberación de Palestina (FDLP) y el Frente Popular para la Liberación de Palestina (FPLP) de George Habash, "no reconocen ya a la dirección de la OLP, pues no representa al pueblo palestino". ${ }^{30}$ La creciente popularidad y poder político del grupo Hamas —en gran parte una creación de Israel- ha constituido el mayor peligro para una pérdida de control de la OLP en los territorios ocupados, principalmente en Gaza. A principios de los 80, Isaac Rabin, entonces Ministro de Defensa, lo promovió como contrapeso a la OLP, pero al final de la década Israel pierde su control, pues dicho grupo adquirió fuerza mediante el apoyo de los palestinos de los territorios ocupados, actuando en contra de los intereses israelíes. ${ }^{31}$ Hamas, con su "línea islámica", ha llamado a la liberación de Palestina, principalmente a través de una constante confrontación con el ejército y colonos judíos. Su éxito dentro de los territorios ocupados se debe principalmente a la desesperación de los palestinos, que comprendieron que la OLP no podía darles una solución. Hamas se volvió un problema

${ }^{30}$ La Jomada, 6/IX/93, p. 48.

31 Ver Wendy Kristianasen Levitt, "De LIslamisme Radical à la Logique Nationaliste", Le Monde Diplomatique, vol. 40, no. 470, mayo 93, p. 25-6; y Milton Viorst, "Learning to Live with the PLO", The Washington Post National Weekly, 19-25/IV/93, p. 25. 
Kenza Elmandjra

tanto para los israelies como para la OLP. El Acuerdo con Israel posiblemente permitiría a la OLP recuperar el poder político perdido frente a Hamas. Con el Acuerdo ha logrado lo que ningún otro grupo, el reconocimiento de Israel y un plan que culminaría en un Estado palestino independiente.

\section{Un camino difícil}

Para entender el significado del Acuerdo en relación al futuro del conflicto y de la región en general, se tiene que considerar el mismo en cuanto a su alcance, sustancia y aplicación, así como la realidad militar y política de la región.

\section{Los límites del acuerdo}

Como se indicó en la primera sección, Israel y la OLP no firmaron un tratado de paz, sino más bien una serie de principios basados principalmente en el mutuo reconocimiento OLP-Israel. En el mismo se aprecian tres ausencias importantes: consideraciones sobre el futuro de las colonias israelíes en los territorios ocupados, la delimitación de fronteras y la cuestión de Jerusalén del Este. Además se pueden identificar varias limitaciones, siendo la más relevante la concerniente al largo proceso de instrumentalización que establece el Acuerdo.

La primera ausencia es sobre el futuro de las colonias judías. Como lo indicó Edward Said, "ni Yasser Arafat, ni sus cercanos compañeros de la OLP que se reunieron con los israelíes en Oslo, han visto con sus ojos lo que es un asentamiento israelin". ${ }^{32}$ Este hecho subraya cierta falta de realismo por parte de los negociadores de la OLP. Existen más de 246 asentamientos israelíes en las colinas y lugares estratégicos de Cisjordania, Gaza y el Golán, pero respecto a su futuro nada fue definido en el Acuerdo de 1993. Como fue indicado en el periódico israelí Haaretz, "las colonias pueden dormir en paz: durante cinco años, el período de autonomía no llevará a ningún cambio". ${ }^{33} \mathrm{Si}$ se aplica el Acuerdo,

${ }^{32}$ Said, "Comment Conjurer ...", p.4.

${ }^{33}$ Haaretz, 7/1X/93, p.3. 
muchos de los asentamientos judíos serán desmantelados. Pero los más importantes, ubicados en puntos estratégicos de Gaza y Cisjordania, no serán tocados debido a que fueron construidos como permanentes. Los asentamientos cubren más del 55\% de los territorios ocupados. En Gaza, por ejemplo, 17 colonias cubren el $30 \%$ del territorio. ${ }^{34}$ Ligadas a la cuestión de los asentamientos, deben ser negociadas cuestiones estratégicas como la distribución del agua y las rutas de comunicación.

La segunda ausencia es la del trazado de fronteras. Como se señaló anteriormente, la OLP y el gobierno de Israel entienden de manera drásticamente diferente en qué consiste la Ciudad de Jericó. La noción palestina es más de 13 veces lo que los israelíes reconocen como superficie de la ciudad. Las fronteras deben ser definidas por el comité mixto palestino-israelí en los próximos cinco años, mediante negociaciones muy difíciles, por la presencia de los asentamientos, por su vínculo con la repartición del agua y por el tema del control de las rutas de comunicación.

La tercera ausencia importante es respecto del futuro de Jerusalén. Haidar Abu Safi, Jefe de la Delegación Palestina en las negociaciones de Washington, cuando el borrador del Acuerdo fue hecho público, declaró: "No puedo aceptar que no haya diálogo sobre el futuro de Jerusalén durante dos años, debemos discutirlo ahora", y añadió que el Acuerdo "está fuera del marco del proceso de paz". ${ }^{35}$ Jerusalén, y más bien Jerusalén del Este, siempre ha sido un importante punto de controversia entre palestinos e isralíes. Israel tomó control de la ciudad después de la guerra de 1967, cuando ocupó Cisjordania. ${ }^{36}$ Existen varias propuestas. Del lado israelí, la solución no es solución, pues no están dispuestos a negociar su control de la Ciudad, que consideran sagrada y que es la capital del Estado. ${ }^{37}$ Esta posición, además resulta justificada

${ }^{34}$ Mohamed Sid Ahmed, "La Métamorphose des Conflits au Proche Orient", Le Monde Diplomatique, Vol. 38, No. 453, dic. 91, p. 13.

${ }_{35}$ La Jomada, 1/IX/93, p. 54.

${ }^{36}$ En Jerusalén del Este se han construido 10 grandes colonias judías donde viven 127.000 colonos, y 5 están en proyecto. En lo que se refiere a la "Jerusalén unificada" viven un total de 500.000 habitantes, de los cuales 361.000 son judíos y 142.600 árabes. Le Monde Diplomatique, vol.39, no.454, enero 92 .

${ }^{37}$ Los Estados Unidos es el único país que tiene su representación diplomática en Jerusalén, los otros la tienen en Tel Aviv. 
Kenza Elmandjra

por intereses estratégicos y de seguridad territorial, dada su posición geográfica en Cisjordania. Por el lado de la OLP, la posición de negociación es que Jerusalén obtenga el estatus de ciudad libre e internacional. La razón fundamental consiste en que es una ciudad sagrada para las tres religiones monoteístas, Judaísmo, Cristianismo e Islam. Esta solución, respaldada por la mayoría de la comunidad internacional (incluido el Vaticano) parece ser la única que presenta un compromiso equitativo entre israelíes y palestinos.

Aparte del alcance limitado del Acuerdo, éste tiene serias restricciones en cuanto a su aplicación. Como lo indicó Rashid Khalidi, Director del Centro de Estudios sobre el Medio Oriente en la Universitad de Chicago y ex-asesor de la delegación palestina en las negociaciones de Washington, el documento presenta varias limitaciones, siendo la más importante relativa al establecimiento de muchas fases intermedias "que complican y limitan sin necesidad el proceso". ${ }^{38}$ Con el fin de llegar a un entendimiento, Israel y la OLP decidieron transferir al futuro los puntos más delicados y firmar más bien un borrador en septiembre de 1993 . Aunque Israel no ha cumplido con sus obligaciones (el retiro de las tropas de seguridad y la transferencia de autoridad a la OLP dentro del tiempo acordado: antes del 13 de diciembre de 1993), cierto es que deberá cumplirlas. Para que se pueda aplicar la primera fase del Acuerdo, Israel y la OLP tienen que llegar a un compromiso sobre tres puntos delicados: la extensión geográfica de Jericó, el control de los puestos fronterizos y la seguridad de las colonias judías en Gaza; las negociaciones prosiguen pero sin resultado alguno. Como lo indicó el Primer Ministro Isaac Rabin, la táctica de negociación israelí es dejar que los palestinos "suden un poco", para obtener más concesiones en estos puntos. 39

${ }^{38}$ Rashid Khalidi, "Blind Curves and Detours on the Road to Self-Rule", The New York Times, 14/IX/93, p. A-25.

${ }^{39}$ The New York Times, 3/1/94, p. A-11. 


\section{Conclusión}

El Acuerdo fue llamado el primer paso en el camino hacia la paz. Pero los caminos por donde israelies y palestinos tienen que caminar parecen desvíos peligrosos. La región sigue estando altamente militarizada, y tanto Israel como la OLP se enfrentan a una creciente oposición interna. Por ejemplo, durante una visita a Washington en noviembre de 1993, el Primer Ministro Isaac Rabin firmó un acuerdo con el Secretario de Defensa norteamericano, Les Aspin, para la compra de veinte aviones de combate F-15 ("Eagle") por 1.800 millones de dólares. ${ }^{40}$ En cuanto a la oposición interna, el gobierno de Isaac Rabin tiene que responder al rechazo de los colonos judíos a aceptar el desmantelamiento de colonias, y la oposición de grupos como Gush Emunim (organización armada de colonos), Shas (partido político extremista ortodoxo) y del partido derechista de oposición, el Likud, encabezado por Benjamin Netanyahu, que se oponen al Acuerdo. La elección de Ehud Omert, del Likud opositor al Acuerdo, como Alcalde de Jerusalén en noviembre de 1993, es un ejemplo de lo que podría resultar, si se llama a nuevas elecciones legislativas. ${ }^{41}$ Por el lado palestino hay que diferenciar entre la OLP y los palestinos de los territorios ocupados. La OLP, con la creciente oposición al Acuerdo se encuentra en plena crisis interna, sobre todo en vista de las dificultades de lograr el cumplimiento de compromisos de Israel. Obviamente, los asesinatos de altos funcionarios de la OLP como Asa'ad Sartawi, cercano colaborador de Arafat, en octubre de 1993, no ayudan. ${ }^{42}$ Además, la organización tiene que enfrentar una creciente oposición en los territorios ocupados por grupos como Hamas, que aumentaría si no se satisfacen las expectativas creadas por el Acuerdo.

El camino hacia la paz es difícil en cualquier instancia, y este Acuerdo, supuestamente iba a abrirlo. Pero si no se respetan sus términos se

${ }^{40}$ El Financiero, 16/XI/93, p. 65.

${ }^{41}$ La Jomada, 4/XI/93, p. 56.

42 Entre septiembre y noviembre además de Sartawi, Mohammad Abu Cha'aban, Maher Ikhail, y Fatah Mounin Shabaytah, fue asesinado el líder del grupo Fatah en Líbano. 
Kenza Elmandjra

volverá más difícil aún. No pueden darse pasos hacia atrás, y los hacia adelante tienen que ser tomados con cuidado y mutuo respeto. Como corolario digamos que los palestinos de los territorios ocupados, hoy pueden izar su bandera casi sin problemas, pero la presencia militar israelí continúa, y la Intifada también. ${ }^{43}$

${ }^{43}$ Desde que se firmó el Acuerdo, de los 12,000 prisioneros políticos palestinos que Israel tiene, solamente 600 fueron liberados. De ellos muchos fueron encarcelados de nuevo. Alain Gersh, "Israéliens et Palestiniens sur un Terrain", Le Monde Diplomatique, vol.41, no.478, en. 94, p. 3. Además, hasta la fecha, la violencia en los territorios ocupados ha cobrado 23 víctimas israelíes y 55 palestinas, The Economist, 22/I/94, p. 43. 